\title{
CONHECIMENTO DE ENFERMEIROS DE UM SERVIÇO DE EMERGÊNCIA SOBRE SEPSE
}

Erick Guilherme Claudino Ferreira ${ }^{1}$

Cassia Regina Vancini Campanharo ${ }^{1}$

Luiz Humberto Piacezzi ${ }^{1}$

Maria Carolina Barbosa Teixeira Lopes Rezende 1

Ruth Ester Assayag Batista ${ }^{1}$

Carla Roberta Monteiro Miura ${ }^{1}$ https://orcid.org/0000-0001-6123-9371

https://orcid.org/0000-0002-7688-2674

https://orcid.org/0000-0001-8855-5630

https://orcid.org/0000-0002-8989-4404

https://orcid.org/0000-0002-6416-1079

https://orcid.org/0000-0003-3528-3568

Objetivo: Avaliar o conhecimento sobre sepse dos enfermeiros de um serviço de emergência. Método: Estudo transversal, quantitativo, desenvolvido em Hospital Universitário, com 41 enfermeiros. Os dados sociodemográficos, de trabalho e de conhecimento sobre sepse foram coletados por meio de instrumentos estruturados e analisados utilizando-se estatística descritiva e inferencial. Resultados: $11(26,83 \%)$ relataram ter tido conteúdo de sepse no treinamento admissional. A maioria das questões obtiveram maior indice de acertos do que erros. As que obtiveram baixo índice de acertos tratavam de definição (2;4,76\%) e do tempo máximo para início da antibioticoterapia (17;40,48\%). Conclusão: Verificou-se desatualização quanto aos protocolos mais recentes sobre definição e gerenciamento da sepse. Assim, este estudo sinaliza a necessidade de medidas institucionais para fixação e atualização deste conteúdo.

Descritores: Enfermeiros; Conhecimento; Sepse; Serviço Hospitalar de Emergência.

\section{NURSES' KNOWLEDGE OF AN EMERGENCY SEPSIS SERVICE}

Objective: To evaluate the knowledge about sepsis of nurses in an emergency service. Method: Cross-sectional, quantitative study, developed at a University Hospital. Involved 41 nurses, the sociodemographic, work and sepsis knowledge data were collected through structured instruments and analyzed using descriptive and inferencial statistics. Results: 11 (26.83\%) reported having sepsis content in admission training. Most of the questions got higher hit rate than errors. Those with low success rates were definition $(2 ; 4.76 \%)$ and maximum time to start antibiotic therapy $(17 ; 40.48 \%)$. Conclusions: Nurses were outdated regarding the most recent protocols on sepsis definition and management. Thus, this study points to the need for institutional measures to consolidate and update this content.

Descriptors: Nurses; Knowledge; Sepsis; Emergency Service, Hospital.

\section{CONOCIMIENTO DE LOS ENFERMEROS DE UN SERVICIO DE SEPSIS DE EMERGENCIA}

Objetivo: Evaluar el conocimiento sobre la sepsis de los enfermeros en un servicio de emergencia. Método: Estudio transversal, cuantitativo, desarrollado en un hospital universitario. Participaron 41 enfermeros, los datos de conocimiento sociodemográfico, laboral y de sepsis se recopilaron a través de instrumentos estructurados e se analizaron mediante estadisticas descriptivas e inferenciales. Resultados: 11 (26.83\%) informaron tener contenido de sepsis en la admisión. La mayoría de las preguntas obtuvieron una mayor tasa de aciertos que los errores. Aquellos con bajas tasas de éxito fueron la definición (2; 4.76\%) y el tiempo para comenzar la terapia con antibióticos (17; 40.48\%). Conclusiones: Los enfermeros estaban desactualizadas con respecto a los protocolos más recientes sobre definición y manejo de sepsis. Este estudio señala la necesidad de medidas institucionales para consolidar y actualizar este contenido.

Descriptores: Enfermeras; Conocimiento; Sepsis; Servicio de Urgencia en Hospital.

${ }^{1}$ Escola Paulista de Enfermagem, Universidade Federal de São Paulo, SP. Brasil.

Autor Correspondente: Carla Roberta Monteiro Miura Email: carla.monteiro@unifesp.br Recebido: 16/12/19 Aceito: 25/7/20 


\section{INTRODUÇÃO}

A sepse é considerada uma importante causa de morbidade e mortalidade. São cerca de 20 a 30 milhões de casos de sepse anualmente em todo o mundo, com altos índices de óbito. No Brasil, ocorrem cerca de 600 mil casos por ano, com 250 mil óbitos, o que corresponde à $16,5 \%$ das causas de óbito do país, caracterizando a sepse como um problema de saúde pública. ${ }^{1,2}$

O SPREAD (Sepsis PREvalence Assessment Database) $)^{(3)}$, um estudo prospectivo de prevalência em 1 único dia, que avaliou a epidemiologia da sepse em Unidades de Terapia Intensiva (UTIs) para adultos, localizadas no Brasil, compreendeu um total de 1.690 UTIs e 19.316 leitos e mostrou uma incidência de sepse nas UTIs de 36,3 casos por mil pacientes- dias e alarmante mortalidade hospitalar de 55,7\%.

A sepse refere-se à uma disfunção orgânica ameaçadora à vida secundária à resposta desregulada do hospedeiro a uma infecção. ${ }^{4,5} \mathrm{O}$ choque séptico é uma anormalidade circulatória e celular/metabólica, secundária a sepse, o suficiente para aumentar significativamente a mortalidade. Requer a presença de hipotensão, com necessidade de vasopressores para manter a pressão arterial média $\geq 65 \mathrm{mmHg}$ e o lactato $\geq 2 \mathrm{mmol} / \mathrm{L}$, após adequada ressuscitação volêmica. $^{2}$

O reconhecimento e tratamento precoces da sepse estão associados a melhores resultados nesses pacientes. Com o propósito de continuar a reduzir a morbidade e mortalidade relacionadas a disfunção orgânica e choque séptico são propostas intervenções conhecidas como pacotes de cuidados para os pacientes com sepse e choque séptico. ${ }^{6,1}$

Para o reconhecimento da sepse é importante a checagem dos sinais vitais, pois alterações dos padrões de normalidade são indicativos de sepse, como alteração da pressão arterial e da saturação de oxigênio. ${ }^{(7)}$ Como o serviço de emergência é, comumente, a porta de entrada onde é realizado o atendimento inicial, e considerando que os profissionais de enfermagem são os primeiros a abordarem esses pacientes, ressalta-se a importância destes profissionais saberem reconhecer os sinais e sintomas sugestivos de sepse e as intervenções a serem realizadas. ${ }^{6,1}$

A partir da suspeita de sepse, o enfermeiro deve contatar a equipe médica, avaliar a necessidade de oxigênio e acesso venoso, e após prescrição médica iniciar o pacote de 1 hora, que inclui coleta de lactato arterial, com resultado apresentado em no máximo 30 minutos; coleta de hemoculturas e culturas de sitios pertinentes, antes da administração de antibiótico, mas sem atrasar o início da terapia antimicrobiana. Coleta de outros exames, que incluem gasometria e lactato arterial, hemograma, creatinina, bilirrubinas e coagulograma. Em até uma hora, e após a coleta das hemoculturas, o médico responsável deve consultar o guia da Comissão de Controle de Infecção Hospitalar (CCIH), prescrever a terapia antimicrobiana, de acordo com os princípios de farmacocinética e farmacodinâmica para otimização da terapia antimicrobiana e notificar o enfermeiro responsável para a administração imediata da medicação. Caso seja descartada a hipótese de sepse, o antimicrobiano pode ser suspenso. ${ }^{2}$

Pacientes hipotensos ou com sinais de hipoperfusão, incluindo hiperlactatemia inicial, devem receber infusão imediata de $30 \mathrm{ml} / \mathrm{kg}$ de cristaloides dentro da $1^{a}$ hora do diagnóstico de hipoperfusão, para dar início a ressuscitação volêmica. Após a realização da reposição volêmica, se o paciente apresentar PAM $<65 \mathrm{mmHg}$, devem ser administrados vasopressores, sendo a Noradrenalina a droga de primeira escolha. Dentro de 2 a 4 horas após o início da ressuscitação volêmica, deve-se solicitar novas doses para os pacientes que apresentarem lactato alterado a partir de duas vezes acima do valor de referência, além de investigar outras causas de hiperlactatemia, que não a hipoperfusão tecidual. ${ }^{2}$

Deve ser realizada a reavaliação das 6 horas em pacientes que apresentem hiperlactatemia, choque séptico ou sinais de hipoperfusão tecidual: deve-se realizar reavaliação da continuidade da ressuscitação volêmica; realizar rapidamente transfusão em pacientes com hemoglobina abaixo de $7 \mathrm{mg} / \mathrm{dl}$ e sinais de hipoperfusão; Monitorar o paciente através de pressão arterial invasiva; monitorizar efeitos de pós carga, evitando um episódio hipertensivo. ${ }^{2}$

Após a identificação da sepse, o enfermeiro responsável deve instituir as intervenções de enfermagem para o paciente, pensando nas especificidades, de acordo com o foco infeccioso e, principalmente, no risco de choque séptico, tendo em vista que o reconhecimento e tratamento precoces estão diretamente relacionados a melhor prognóstico. Dentre as principais intervenções de enfermagem destacam- se a monitorização hemodinâmica, respiratória e do nivel de consciência. ${ }^{2}$ 
Estudo que avaliou a implementação de cuidados direcionados por enfermeiros para promover a identificação oportuna e o tratamento precoce da sepse no serviço de emergência, mostrou que a atuação do enfermeiro é capaz de melhorar a adesão aos pacotes de cuidado e reduzir as taxas de mortalidade relacionadas à sepse no hospital. ${ }^{8}$ Por outro lado, estudo que avaliou o conhecimento da equipe de enfermagem mostrou que muitos profissionais, apesar de saberem algo sobre sepse, estão desatualizados, o que prejudica a identificação desta condição. Além disso, muitos profissionais, também, não sabem quais condutas devem ser tomadas após o diagnóstico. ${ }^{(6,1)}$

Em reconhecimento a magnitude do problema que a sepse representa, e tendo em vista a importância do reconhecimento e da aplicação dos protocolos de sepse precocemente, é fundamental a avaliação do conhecimento dos profissionais de enfermagem sobre o assunto. A partir da identificação de possíveis lacunas no conhecimento destes profissionais sobre o tema, intervenções específicas podem ser implementadas o que pode melhorar o processo assistencial e os desfechos desses indivíduos.

\section{OBJETIVO}

Estudo teve como objetivo avaliar o conhecimento sobre sepse dos enfermeiros de um serviço de emergência de um hospital público de grande porte.

\section{MÉTODO}

\section{Tipo de estudo}

Estudo transversal e analítico do tipo quantitativo.

\section{Local do estudo}

A pesquisa foi desenvolvida em hospital universitário de grande porte da cidade de São Paulo.

\section{Participantes do estudo}

Fizeram parte deste estudo, 41 enfermeiros do serviço de emergência do referido hospital, de todos os turnos de trabalho, que voluntariamente concordaram em participar da pesquisa assinando um termo de consentimento informado.

Foram excluidos aqueles que estiveram ausentes, por férias ou licença, no período da coleta de dados, além dos enfermeiros que se recusaram a participar da pesquisa, totalizando sete enfermeiros excluídos do estudo.

\section{Coleta de dados}

O pesquisador compareceu na unidade de emergência, nos diferentes turnos de trabalho, e convidou os trabalhadores a participarem da pesquisa e responderem aos questionários entre os meses de agosto e setembro de 2019.

Os dados sociodemográficos e de trabalho foram coletados por meio de um instrumento estruturado elaborado pelos pesquisadores, e o conhecimento dos enfermeiros sobre sepse foi verificado por meio de outro instrumento estruturado, criado pelos pesquisadores com base na literatura recente sobre o assunto, que trata-se, do Consenso Internacional de Definições Sepse-3 $3^{3}$, e o protocolo de gerenciamento de sepse do Instituto Latino Americano de Sep$\mathrm{se}^{(7)}$. O instrumento foi composto por 14 questões objetivas, que abordaram os seguintes assuntos relacionados à sepse: sinais e sintomas, diagnóstico, tratamento e complicações. O questionário foi aplicado sob observação direta do pesquisador, evitando eventuais consultas, não havendo limite de tempo para o completo preenchimento do questionário.

\section{Análise e tratamento dos dados}

Após o término da coleta, os dados foram agrupados em planilha do programa Microsoft Excel versão 2016, e apresentados em forma de gráficos e tabelas utilizando-se a estatística descritiva e inferencial.

\section{Aspectos éticos}

Os dados foram coletados mediante aprovação do Comitê da Ética da Universidade Federal de São Paulo (Parecer $n \cong$ 3.346.040/CAAE $n=12625219.8 .0000 .5505)$ e assinatura do termo de consentimento livre e esclarecido pelos sujeitos da pesquisa.

\section{RESULTADOS}

\section{Dados sociodemográficos e de trabalho}

Os sujeitos da pesquisa tinham idade média de 32 anos, variando de 21 a 50 anos, 28 (68,29\%) eram do sexo masculino, 23 (56,10\%) da cor branca, $23(57,5 \%)$ de estado civil solteiro, $31(77,5 \%)$ residentes na região Sudeste, sendo $12(30,77 \%)$ procedentes da cidade de São Paulo, 31(75,61\%) deles tinham cursado pós-graduação, conforme mostra a Tabela 1. 
Tabela 1 - Caracterização dos enfermeiros do serviço de emergência segundo variáveis sociodemográficas. São Paulo, 2019.

\begin{tabular}{|c|c|c|c|c|c|}
\hline Variável & Categoria & $\mathbf{n}$ & $\%$ & média & $(\min -\max )$ \\
\hline \multirow[t]{2}{*}{ Idade } & - & - & - & 32 & $21-50$ \\
\hline & masculino & 13 & 31,71 & - & - \\
\hline \multirow[t]{4}{*}{ Gênero } & feminino & 28 & 68,29 & - & - \\
\hline & outro & 0 & 0 & - & - \\
\hline & Norte & 2 & 5 & - & - \\
\hline & Nordeste & 4 & 10 & - & - \\
\hline \multirow[t]{4}{*}{ naturalidade } & Sul & 2 & 5 & - & - \\
\hline & Sudeste & 31 & 77,5 & - & - \\
\hline & Centro-oeste & 1 & 2,5 & - & - \\
\hline & Branca & 23 & 56,10 & - & - \\
\hline \multirow[t]{6}{*}{ Cor } & Parda & 11 & 26,83 & - & - \\
\hline & Preta & 5 & 12,20 & - & - \\
\hline & Indígena & 0 & 0 & - & - \\
\hline & amarela & 2 & 4,88 & - & - \\
\hline & Solteiro & 23 & 57,50 & - & - \\
\hline & União estável & 3 & 7,50 & - & - \\
\hline \multirow[t]{4}{*}{ estado civil } & casado & 10 & 25 & - & - \\
\hline & divorciado/separado & 4 & 10 & - & - \\
\hline & viúvo & 0 & 0 & - & - \\
\hline & Superior & 10 & 24,39 & - & - \\
\hline escolaridade & Pós-graduação & 31 & 75,61 & - & - \\
\hline
\end{tabular}

Dentre os sujeitos, $17(41,46 \%)$ eram do turno da noite e 16 (39,02\%) da noite, tinham em média 5,31 anos de formados, com tempo médio de atuação na enfermagem de 4,43 anos e de 2,94 anos no serviço emergência, variando de um mês a 13 anos.

Tabela 2 - Caracterização dos enferme iros do serviço de emergência segundo variáveis de trabalho. São Paulo, 2019.

\begin{tabular}{|c|c|c|c|c|c|}
\hline Variável & Categoria & $\mathbf{n}$ & $\%$ & $\begin{array}{l}\text { Média } \\
\text { (anos) }\end{array}$ & $\begin{array}{c}\min -\max \\
\text { (anos) }\end{array}$ \\
\hline Tempo de formado & - & - & - & 5,31 & $0,5-14$ \\
\hline Tempo de atuação & - & - & - & 4,43 & $0,08-13$ \\
\hline \multirow[t]{2}{*}{ Tempo de atuação na emergência } & - & - & - & 2,94 & $0,08-13$ \\
\hline & manhã & 8 & 19,51 & - & - \\
\hline \multirow[t]{2}{*}{ Turno de trabalho } & tarde & 16 & 39,02 & - & - \\
\hline & noite & 17 & 41,46 & - & - \\
\hline
\end{tabular}




\section{Contato prévio com o conteúdo de sepse}

Dos sujeitos participantes da pesquisa, 39 (95,12\%) referiram ter tido o conteúdo de sepse na graduação, sendo que numa escala variando entre excelente, muito bom, bom, regular e ruim, 16 (41,03\%) avaliou o conteúdo recebido como na graduação como sendo "bom".

Entre aqueles com nivel de pós-graduação, 25
(62,50\%) referiram ter tido o conteúdo de sepse no curso e entre aqueles que avaliaram o conteúdo, a maioria classificou - o como "excelente", 9 (34,62\%).

Onze dos enfermeiros (26,83\%), referiram ter tido esse conteúdo no treinamento admissional, sendo que o conteúdo do treinamento foi considerado como "bom" por 6 deles $(46,15 \%)$.

Tabela 3 - Caracterização dos enfermeiros do serviço de emergência segundo contato prévio com o conteúdo de sepse. São Paulo, 2019.

\begin{tabular}{|c|c|c|c|}
\hline Variável & Categoria & $\mathrm{n}$ & $\%$ \\
\hline \multirow[t]{2}{*}{ Conteúdo de sepse na graduação } & Sim & 39 & 95,12 \\
\hline & Não & 2 & 4,88 \\
\hline \multirow[t]{2}{*}{ Conteúdo de sepse na pós-graduação } & $\begin{array}{l}\text { Sim } \\
\text { Não }\end{array}$ & $\begin{array}{c}25 \\
4\end{array}$ & $\begin{array}{c}62,50 \\
10\end{array}$ \\
\hline & Não se aplica & 11 & 27,50 \\
\hline \multirow[t]{2}{*}{ Conteúdo de sepse em treinamento admissional } & Sim & 11 & 26,83 \\
\hline & Não & 30 & 73,17 \\
\hline
\end{tabular}

\section{Conhecimento sobre sepse}

Ao avaliar as respostas do questionário para avaliação do conhecimento sobre sepse, observou-se que a média de acertos por profissional foi de 8,6 (61,90\%) de 14 , variando de $5(35,71 \%)$ até 13 (92,86\%) acertos, conforme mostra a Figura 1.

Figura 1. - Número de acertos por questão. São Paulo, 2019.

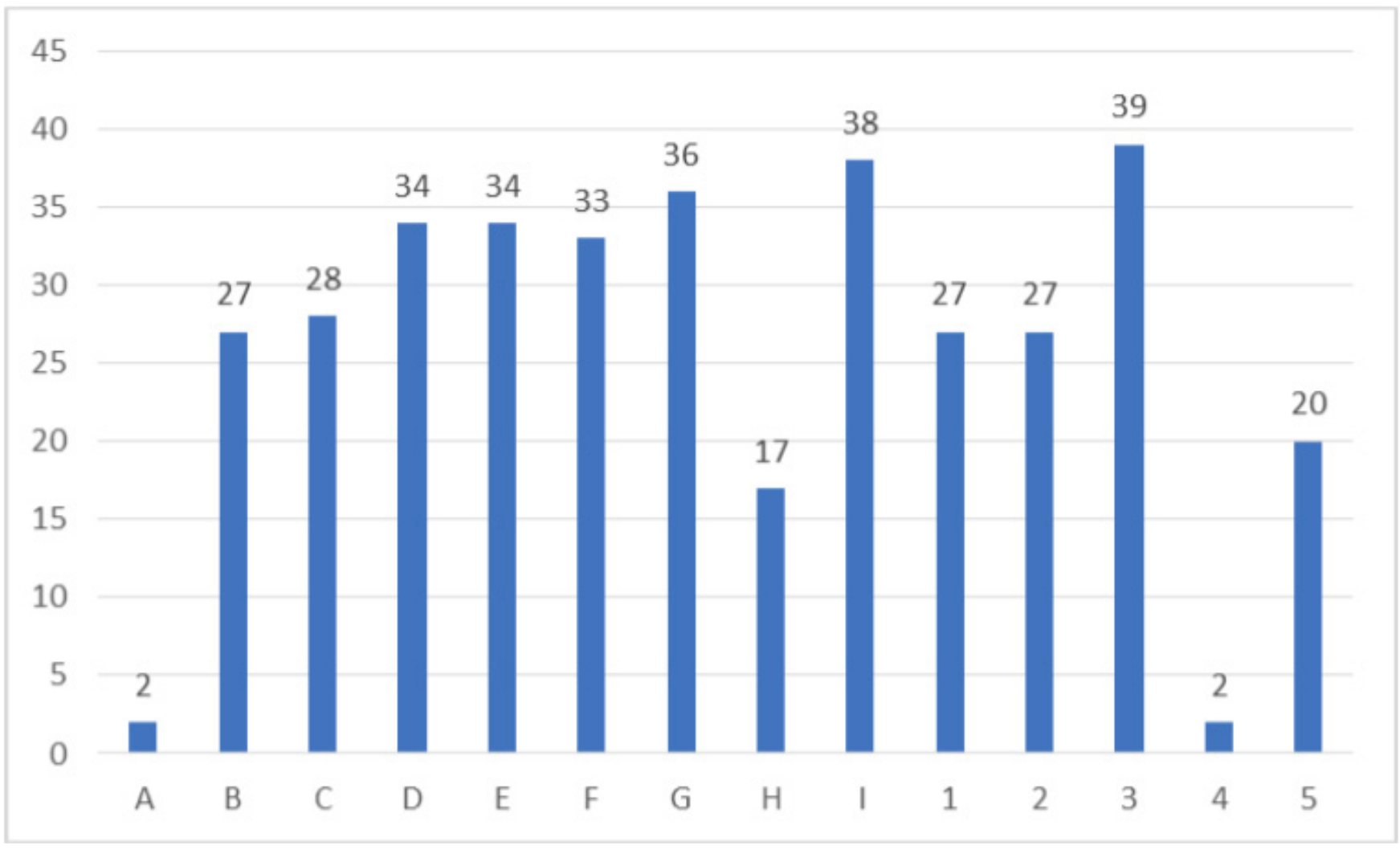


A maioria das questões obteve maior índice de acertos do que erros. A questão que obteve maior número de acertos (39; 92,86\%) versava sobre diagnóstico diferencial entre Sepse, Síndrome de resposta inflamatória sistêmica, Choque séptico e suspeita de infecção do trato urinário.

Duas questões apresentaram índices muito baixos de acerto (2; 4,76\%), uma delas sobre a definição de sepse e outra sobre diagnóstico diferencial entre Sepse, Síndrome de resposta inflamatória sistêmica, Choque séptico e Osteomielite, sendo que 22 (52,38\%) de 41 indivíduos, selecionou "Síndrome de resposta inflamatória sistêmica" como sendo o diagnóstico compatível para o caso descrito, quando a resposta correta era Sepse.

Apenas 17 (40,48\%), acertaram a questão referente ao tempo máximo indicado para início da antibioticoterapia.

\section{DISCUSSÃO}

Considerando os resultados da pesquisa, em que 95,12\% dos entrevistados receberam o conteúdo de sepse durante a graduação e, dentre eles, 62,5\% o receberam novamente durante o curso de pós-graduação, o resultado da presente pesquisa apresenta uma média de acertos aquém do esperado, $61,9 \%$.

Essa média de acertos pode ser influenciada por uma não continuidade da educação fora do ambiente acadêmico, aonde apenas $26,83 \%$ dos entrevistados disseram ter recebido o conteúdo de sepse em seu treinamento admissional. Estudo que mostrou uma desatualização dos enfermeiros quanto ao conteúdo de sepse também sinalizou que apenas 16,6\% dos profissionais receberam treinamentos em serviço sobre o tema e $10 \%$ dos entrevistados conheciam algum protocolo clínico de gerenciamento de sepse ${ }^{9}$

Os sujeitos da pesquisa tiveram um baixo índice de acertos sobre o tempo de início da antibioticoterapia e sobre o diagnóstico de sepse. Esse achado sinaliza uma falta de atualização quanto a nova definição de sepse do Sepsis-3느, bem como quanto ao protocolo de gerenciamento de sepse do Instituto Latino-Americano de Sepse ${ }^{7}$, que tratam -se de orientações mundiais, e portanto, devem ser de conhecimento dos profissionais atuantes. Dados esses que sinalizam para a necessidade de que este tema seja abordado no ambiente profissional, tanto na admissão, quanto em programas institucionais, por meio da educação continuada.

Um estudo australiano ${ }^{10}$, também envolvendo enfermeiros do setor de emergência, igualmente mostrou conhecimento deficiente quanto aos critérios diagnósticos e indicadores clínicos de identificação da sepse.

Estudo ${ }^{11}$ que buscou verificar a aplicação do algoritmo da sepse por enfermeiros atuantes em Unidade de Terapia In- tensiva situada no Vale do Paraíba, evidenciou que 55\% dos enfermeiros não souberam responder a sequência correta do atendimento ao paciente com sepse nas primeiras seis horas, apenas 65\% apontaram corretamente o momento da realização de culturas, quanto as terapias de suporte no tratamento da sepse, 55\% selecionaram a afirmativa incorreta e apenas $5 \%$ soube informar sobre o tempo de redução da dosagem do lactato.

O profissional enfermeiro, sem um suporte educacional de longo prazo, tende a não se atualizar autonomamente, ficando desatualizado sobre as novas diretrizes, protocolos e definições. Isto fica comprovado em diversos estudos realizados, tanto sobre sepse ${ }^{11,12,13}$, quanto sobre outras temáticas de atuação do enfermeiro ${ }^{14}$, em estudos desenvolvidos no Brasil e em demais nacionalidades ${ }^{15}$. Como fatores que influenciam negativamente na participação de profissionais de enfermagem na educação permanente, pode-se citar o quantitativo do pessoal de enfermagem e o horário das atividades. ${ }^{16}$

A instauração de medidas de educação permanente, impactam positivamente no nível de conhecimento, na prática e na gestão do cuidado, como mostra o resultado de outras pesquisas realizadas que avaliaram o conhecimento do enfermeiro após receber algum tipo de treinamento educacional, como por exemplo, uma pesquisa ${ }^{17}$, que avaliou o impacto da implementação do protocolo de sepse, quanto ao tempo para administração inicial de antibióticos e mortalidade hospitalar, que mostrou significativa melhora após implantação do treinamento.

Outro estudo ${ }^{18}$, também desenvolvido com enfermeiros do setor de emergência, mostrou que após algumas intervenções, dentre elas uma intervenção educativa, houve redução do tempo de implementação do pacote de sepse, assim como o tempo médio de administração do antibiótico também diminuiu, além de uma queda de 5,9\% da mortalidade geral por sepse, quando se comparou dados pré e pós intervenção.

No processo de educação dos profissionais da saúde, entende-se como educação permanente qualquer tipo de atividade de capacitação caracterizado pela relação com o processo de trabalho institucional.

Uma prática pedagógica motivadora e interativa utilizada no treinamento de profissionais, pode ser um excelente meio de motiva -los a transformar a realidade em que estão inseridos e implementar cuidados seguros e baseados em evidências científicas. As metodologias interativas educacionais se identificam com o conceito contemporâneo de ensino, que considera o aprendiz como o centro do processo e construtor da sua aprendizagem, capaz de resolver problemas em situações muito próximas às reais e com a possibilidade de avaliação formativa e continuada. 
Estudos nacionais ${ }^{19}$ e internacionais ${ }^{20,21}$ têm mostrado que o uso da simulação clínica no treinamento de enfermeiros é capaz de promover aumento significativo da confiança, autoeficácia e do julgamento clínico acerca do reconhecimento precoce e manejo da sepse, culminando com a melhora dos desfechos dos pacientes.

Dentro deste contexto, a Simulação realística, especificamente a simulação de alta fidelidade, mostra -se como uma excelente metodologia para aquisição de habilidades e competências que tem adquirido especial destaque na educação em saúde, atendendo à crescente preocupação com a segurança do paciente e profissional ${ }^{19,22}$, e portanto sugerimos seu emprego na educação continuada de enfermeiros, com o intuito de aumentar o índice de desfechos positivos dos pacientes em relação à sepse.

\section{Limitações do estudo}

O presente estudo apresenta a limitação de ter sido realizado apenas com enfermeiros de um único setor hospitalar, de um único hospital, em apenas um país. Apresenta também a limitação de ter utilizado um questionário autoral, que, no entanto, poderá ser utilizado em novas pesquisas deste tema, aumentando o valor atribuído ao mesmo.

\section{Contribuições para a prática}

Apesar das limitações descritas, o presente estudo é importante na medida em que contribuiu para o corpo de conhecimento sobre como os enfermeiros de emergência reconhecem e respondem a pacientes com sepse, além disso, corrobora outros estudos que apontam para a necessidade de medidas de educação permanente contínuas, com vistas ao aprimoramento profissional, resultando em um atendimento mais eficiente, seguro e com melhor gestão do cuidado.

\section{CONCLUSÃO}

Este estudo mostrou que os profissionais enfermeiros sujeitos da pesquisa possuem conhecimento sobre sepse, porém com conteúdos desatualizados, especialmente no que se refere às novas definições e diretrizes de atendimento à sepse, o que é alarmante, haja visto que o enfermeiro do serviço de emergência representa uma das primeiras linhas de defesa de um paciente contra a sepse, através do reconhecimento precoce e abrangente, bem como por meio do conhecimento de planos de tratamento atuais e baseados em evidências.

Apoiando-se no pressuposto pedagógico de discussão da realidade com base nos elementos que façam sentido para os sujeitos envolvidos, sugerimos o uso de metodologias ativas de aprendizagem, dentre algumas delas, ganha destaque a simulação clínica de alta fidelidade, onde os profissionais, por meio de cenários simulados criados a partir da sua realidade, podem desenvolver o pensamento crítico e treinamento dos mais atuais protocolos sem risco de danos a eles mesmos ou pacientes.

Contribuições dos Autores: Erick Guilherme Claudino Ferreira e Cassia Regina Vancini Campanharo responsáveis por todas as etapas do estudo assegurando as questões de precisão ou integridade de qualquer parte; Luiz Humberto Piacezzi, contribuições substanciais para o desenho do estudo, análise e interpretação de dados e elaboração e revisão crítica do conteúdo intelectual do estudo; Maria Carolina Barbosa Teixeira Lopes Rezende e Ruth Ester Assayag Batista, contribuições substanciais para a aquisição e interpretação de dados do estudo e aprovação da versão final a ser publicado; Carla Roberta Monteiro Miura, responsável por todos os aspectos do estudo, assegurando as questões de precisão ou integridade de qualquer parte.

\section{REFERÊNCIAS}

1. Melech CS, Paganini MC. Avaliação do conhecimento de médicos e equipe de enfermagem nas ocorrências de sepse. Revista Médica da UFPR. 2015 [citado em 2018 dez. 20]; 3(3):127-132. Disponível em: https://revistas.ufpr.br/ revmedicaufpr/article/view/47544.

2. Viana RAPP, Machado FR, Souza JLA. Sepse, um problema de saúde pública: a atuação e colaboração da enfermagem na rápida identificação e tratamento da doença. São Paulo: COREN-SP, 2017 [citado em 2018 dez. 20] Disponivel em: http://www.ilas.org.br/assets/arquivos/ferramentas/livro-sepse-um-problema-de-saude-publica-coren-ilas.pdf.

3. Machado FR, Cavalcanti AB, Bozza FA, Ferreira EM, Angotti Carrara FS, Sousa JL et al. The epidemiology of sepsis in Brazilian intensive care units (the Sepsis PREvalence Assessment Database, SPREAD): an observational study. Lancet Infect Dis. 2017 [citado em 2018 dez. 20];17(11):1180-9. Disponivel em:https://www.sciencedirect.com/science/ article/pii/S1473309917303225?via\%3Dihub

4. Singer M, Deutschman CS, Seymour CW, Shankar-Hari M, Annane D, Bauer M, et al. The Third International Consensus Definitions for Sepsis and Septic Shock (Sepsis-3). JAMA [Internet]. 2016 Feb; [citado em 2019 fev. 20]; 315(8):801-10. Disponivel em: https://doi.org/10.1001/jama.2016.0287 
5. Shankar-Hari M, Phillips GS, Levy ML, Seymour CW, Liu VX, Deustschman CS et al. Sepsis Definitions Task Force. Developing a New Definition and Assessing New Clinical Criteria for Septic Shock: For the Third International Consensus Definitions for Sepsis and Septic Shock (Sepsis-3); [online]; JAMA. 2016 [citado em 2018 dez. 20]; 315(8):77587. Disponivel em: https://jamanetwork.com/journals/jama/fullarticle/2492876.

6. Pinheiro WR, Lopes KRF, Akerman M, Batista HMT, Bernardo GP, Bernardo LP et al. Grau de conhecimento dos profissionais de saúde sobre o protocolo de sepse nas UTI's e nas unidades de emergência do Cariri, Ceará, Brasil; Biomedical \& Health Sciences [Internet]. 2017 [Citado em 2018 dez. 20]. 1(1):18-28. Disponivel em: https://studylibpt. com/doc/3991553/grau-de-conhecimento-dos-profissionais-de-sa\%C3\%BAde-sobre-o-p..

7. Instituto Latino-Americano de Sepse. Implementação de protocolo gerenciado de sepse: protocolo clínico. Atendimento ao paciente adulto com sepse/choque séptico [Internet]. 2017 Jun; [citado em 2019 fev. 20]. Available from: https://www.ilas.org.br/assets/arquivos/ferramentas/protocolo-detratamento.pdf

8. Ferquson A, Coates DE, Osborn S, Blackmore CC, Williamns B. Early Nurse-Directed Sepsis Care. Am J. Nurs 2019 [citado em 2019 out. 27]; 119(1): 52-58. Disponivel em: https://www.ncbi.nlm.nih.gov/pubmed/30589710

9. Goulart LS, Junior MAF, Sarti ECFB, et al. Os enfermeiros estão atualizados para o manejo adequado do paciente com sepse? Escola Anna Nery [internet]. 2019 Jan [Citado em 2019 set. 23]; 23(4):e20190013. Disponivel em: http:// www.scielo.br/scielo.php?script=sci_arttextEpid=S1414-81452019000400208\&lng=pt\&nrm=iso\&tlng=pt.

10. Harley A, Jonhnston ANB, Denny KJ, Keijzers G, Crilli J, Massey D. Emergency nurses knowledge and understanding of their role in recognising and responding to patients with sepsis: a qualitative study. Int Emerg Nurs. 2019 [citado em 2019 set. 23];43:106-12. Disponivel em: https://www.researchgate.net/publication/330858742_Emergency_nurses'_knowledge_and_understanding_of_their_role_in_recognising_and_responding_to_patients_with_ sepsis_A_qualitative_study

11. Peninck PP, Machado RC. Aplicação do algoritimo da sepse por enfermeiros na Unidade de Terapia Intensiva. Rev Rene 2012 [citado em 2019 out. 27];13 (1):187-99.Disponivel em: http://www.periodicos.ufc.br/rene/article/view/3793

12. Almeida APSR, Belchior PK, Lima MG, Souza LP. Conhecimento do profissional enfermeiro a respeito da sepse. BJSCR. 2013 [Citado em 2019 set. 23] 4(4):05-10. Disponivel em: https://www.mastereditora.com.br/bjscr.

13. Delaney MM, Friedman MI, Fitzpatrick JJ. Impact of a Sepsis Educational Program on Nurse Competence. J Contin Educ Nurs. 2015 [Citado em 2019 set. 23] 46(4):179-186. Disponivel em: https://www.healio.com/nursing/journals/ jcen/2015-4-46-4/\%7B682b52b2-aaae-4d4c-ac55-96019053e58e\%7D/impact-of-a-sepsis-educational-program-on-nurse-competence.

14. Nascimento RAM, Assunção MSC, Junior JMS, Amendola CP, et al. Conhecimento do enfermeiro para identificação precoce da injúria Renal Aguda. Rev. Esc. Enferm.USP. 2016 [Citado em 2019 set. 23] 50(3):399-404. Disponivel em: http://www.scielo.br/scielo.php?script=sci_arttextEpid=S008062342016000300399\&lng=en\&tlng=en.

15. Storozhuk AS, MacLeod MLP, Freeman S, Banner D. A survey of sepsis knowledge among Canadian emergency department registered nurses. Australias. Emerg. Care. 2019 Jun [Citado em 2019 Set. 23] 22(2):119-125. Disponivel em: https://www.sciencedirect.com/science/article/abs/pii/S2588994X19300077?via\%3Dihub.

16. Tibola TSA, Cordeiro ALP de C, Stacciarini TSG, Engel RH, Costa DG, Haas VJ. Fatores que influenciam a participação dos profissionais de Enfermagem na Educação Permanente em Hospital Público. Enferm Foco [Internet] 2019 [Citado em 2020 Jul. 15] 10(2):11-16. Disponivel em: http://revista.cofen.gov.br/index.php/enfermagem/article/ view/2044/532.

17. Bruce HR, Maiden J, Fedullo PF, Kim SC. Impact of Nurse-Initiated ED Sepsis Protocol on Compliance With Sepsis Bundles, Time to Initial Antibiotic Administration, and In-Hospital Mortality. J Emerg Nurs. 2015 Mar [Citado em 2019 Set. 23] 41(2): 130-137. Disponivel em: https:// jenonline.org/article/S0099-1767(14)00584-4/fulltext www.

18. Threatt DL. Improving Sepsis Bundle Implementation Times: A Nursing Process Improvement Approach. J Nurs Care Qual. 2019 [Citado em 2019 Set. 26]doi: 10.1097/NCQ.0000000000000430. Disponivel em: https://www.ncbi. nlm.nih.gov/pubmed/31290779

19. Davis AH, Hayes SP. Simulation to Manage the Septic Patient in the Intensive Care Unit. Crit Care Nurs Clin North Am. 2018 Set [Citado em 2019 Set. 23] 30(3):363-377. Disponivel em: https://www.sciencedirect.com/science/article/pii/S0899588518309523?via\%3Dihub.

20. Carvalho LR. Julgamento clínico e autoeficácia de enfermeiros para o manejo da sepse: uso da simulação clínica. [Tese]. São Paulo: Universidade Federal de São Carlos;2018.

21. Herron JBT, Harbit A, Dunban JAT. Subduing the killer - sepsis; through simulation. BMJ Evid Based Med. 2019 [Citado em 2019 Set. 27];24 (1):26-9. Disponivel em: https://ebm.bmj.com/content/24/1/26.long

22. Pazin Filho A, Scarpelini S. Simulação: definição. Medicina (Ribeirão Preto). 2007 [Citado em 2019 Set. 24] 40(2):1626. Disponivel em: http://revista.fmrp.usp.br/2007/vol40n2/2_simulacao_definicao.pdf 